BMJ Open Ophthalmology

\title{
Monocular and binocular visual impairment in the UK Biobank study: prevalence, associations and diagnoses
}

\author{
Martin McKibbin, ${ }^{1}$ Tracey M Farragher, ${ }^{2}$ Darren Shickle, ${ }^{2}$ on behalf of UK Biobank \\ Eye and Vision Consortium
}

To cite: McKibbin M, Farragher TM, Shickle D, et al. Monocular and binocular visual impairment in the UK Biobank study: prevalence, associations and diagnoses. BMJ Open Ophthalmology 2018;3:e000076. doi:10.1136/ bmjophth-2017-000076

- Additional material is published online only. To view please visit the journal online (http://dx.doi.org/10.1136/ bmjophth-2017-000076).

Received 17 February 2017 Revised 4 September 2017 Accepted 4 January 2018
Check for updates

${ }^{1}$ Eye Clinic, Leeds Teaching Hospitals NHS Trust, Leeds, UK ${ }^{2}$ Academic Unit of Public Health, University of Leeds, Leeds, UK

Correspondence to Martin McKibbin; martin. mckibbin@nhs.net

\section{ABSTRACT}

Objective To determine the prevalence of, associations with and diagnoses leading to mild visual impairment or worse (logMAR $>0.3)$ in middle-aged adults in the UK Biobank study.

Methods and analysis Prevalence estimates for monocular and binocular visual impairment were determined for the UK Biobank participants with fundus photographs and spectral domain optical coherence tomography images. Associations with socioeconomic, biometric, lifestyle and medical variables were investigated for cases with visual impairment and matched controls, using multinomial logistic regression models. Self-reported eye history and image grading results were used to identify the primary diagnoses leading to visual impairment for a sample of $25 \%$ of cases.

Results For the 65033 UK Biobank participants, aged 40-69 years and with fundus images, $6682(10.3 \%)$ and $1677(2.6 \%)$ had mild visual impairment or worse in one or both eyes, respectively. Increasing deprivation, age and ethnicity were independently associated with both monocular and binocular visual impairment. No primary diagnosis for the recorded level of visual impairment could be identified for $49.8 \%$ of eyes. The most common identifiable diagnoses leading to visual impairment were cataract, amblyopia, uncorrected refractive error and vitreoretinal interface abnormalities.

Conclusions The prevalence of visual impairment in the UK Biobank study cohort is lower than for population-based studies from other industrialised countries. Monocular and binocular visual impairment are associated with increasing deprivation, age and ethnicity. The UK Biobank dataset does not allow confident identification of the causes of visual impairment, and the results may not be applicable to the wider UK population.

\section{INTRODUCTION}

At present, data on the prevalence, associations with and diagnoses leading to visual impairment among middle-aged adults in the UK, especially those of working age, are lacking. Reliable prevalence data do exist but mainly for children and the elderly or for those with certifiable visual impairment. For middle-aged adults, the most widely used data come from a number of international, population-based studies, many of

\section{Key messages}

What is already known about this subject? Current and reliable data on the prevalence of, associations with and diagnoses leading to monocular and binocular impairment is lacking, particularly for middle-aged adults.

\section{What are the new findings?}

In this study with UK Biobank participants, the prevalence of monocular and binocular visual impairments was $10.3 \%$ and $2.6 \%$, respectively. Increasing Townsend deprivation score, age and ethnicity were independently associated with both monocular and binocular visual impairments. Uncorrected refractive error, cataract, amblyopia and vitreoretinal interface abnormalities were the common identifiable causes of visual impairment. Despite the use of self-reported eye history, fundus photographs and spectral domain optical coherence tomography images, no identifiable cause for the recorded level of visual impairment was found in almost half the eyes.

How might these results change the focus of research or clinical practice?

The UK Biobank dataset does not allow confident identification of the primary causes of visual impairment. However, most of the visual impairment in middle-age with an identifiable cause would be amenable to treatment. Despite the large number of participants, results from the dataset may still not be applicable to the wider UK population. Future research should explore the reasons behind the association of visual impairment with deprivation and ethnicity.

which concluded almost 20 years ago. ${ }^{1}$ Given the recent increases in obesity and diabetes mellitus and national differences in ethnicity, smoking history and socioeconomic status, the findings of these studies may not be relevant to the current UK population.

UK Biobank is a voluntary, cross-sectional study of UK residents that aims to investigate how lifestyle, environment and genes influence the health of adults, aged 40-69 years, in the UK. ${ }^{2}$ The size and breadth of the UK Biobank dataset provides a unique 
opportunity to collect accurate data on the prevalence and causes of visual impairment among participants. The aims of this project were to determine the prevalence of monocular and binocular visual impairment among UK Biobank participants, to investigate associations with known and potential socioeconomic, biometric, lifestyle and medical variables and to determine the causes of visual impairment among UK Biobank participants using self-reported eye history and image grading.

\section{MATERIALS AND METHODS}

\section{Study population}

Over the course of 2006-2010, UK Biobank recruited 503325 participants at 22 centres by postal invitation $\left(5.47 \%\right.$ response rate). ${ }^{2}$ Data collection during the baseline and enhanced assessments has been reported elsewhere. ${ }^{3-5}$ Deprivation was assessed using the Townsend score: a measure of deprivation for each postcode area, derived from census data on employment, household overcrowding, car and home ownership.

Additional enhancements to the baseline assessment visit were included for the collection of eye data from 2009. This included visual acuity with habitual correction, using a semiautomated system using logMAR optotypes, autorefraction and self-reported eye history for 133668 participants, of whom 65033 also underwent colour fundus photography and spectral domain optical coherence tomography (OCT) imaging. From these 65033 UK Biobank participants with imaging, 8359 cases had mild visual impairment or worse (logMAR $>0.3$ or Snellen $>6 / 12$ ) in at least one eye and were matched with controls, without visual impairment, by age (5year bands), sex and ethnic group at a ratio of 1:4.

In a nested pilot study, the ability to identify both the primary cause of and associations with visual impairment, using self-reported eye history and image grading, was investigated in a subset of $25 \%$ of those with visual impairment and fundus imaging. Cases for the nested pilot study were selected in order to be representative of all participants with visual impairment in terms of visual acuity, age, sex and ethnicity.

The UK Biobank project received approval from the North-West Multicentre Research Ethics Committee. Approval was also obtained for access to anonymised UK Biobank data by researchers, without the need for additional approvals.

\section{Baseline and enhanced assessments}

Data collection during the baseline and enhanced assessments has been reported elsewhere. ${ }^{3-5}$ In brief and of relevance to the eye, this comprised a history of spectacle or contact lens wear, eye surgery and disease, logMAR acuity, refractive error and fundus imaging. Using the Topcon 3D-OCT 1000 Mark 2 (Topcon Corporation, Tokyo, Japan), a single non-mydriatic, $45^{\circ}$ digital, colour image, centred on the fovea, and a spectral domain OCT cube scan of the macula, covering a $6 \mathrm{~mm} * 6 \mathrm{~mm}$ retinal area (128 horizontal line scans comprised of 512 A-scans) were captured for both eyes.

\section{Analysis of fundus photographs and OCT images}

Images were analysed using the Topcon OCT viewer software (V.4.21, Topcon Corporation) by experienced clinicians or by non-medical graders, certified for the detection and classification of diabetic retinopathy by the National Health Service (NHS) Diabetes Eye Screening Programme. Fundus photographs were assessed for image quality, media opacity, optic disc or retinal vascular or other abnormalities and the presence of signs of age-related maculopathy. OCT images were assessed for image quality, vitreoretinal interface abnormalities (VRIA) and intraretinal abnormalities. The presence or absence of ocular abnormalities and disease was confirmed using standard definitions. ${ }^{6-10}$ Image quality grades are defined in online supplementary table 1 . The main reason for adequate or inadequate images was assessed as being either technical or the result of media opacity.

Assigning the primary diagnosis leading to visual impairment Using the results of image grading and the level of visual impairment, together with self-reported eye history, graders assigned the primary diagnosis leading to visual impairment for each eye in a sample of $25 \%$ of the cases. (These cases were selected in order to be representative of the larger group of all participants with visual impairment in terms of the degree of visual impairment, age, sex and ethnicity.) For eyes in which no cause was identified, the presence or absence of habitual correction at the time visual acuity was recorded, and the refractive errors were noted. For the eyes with unaided visual impairment, uncorrected refractive error was considered to be the cause of visual impairment when no other pathology was identified and there was a spherical equivalent of $\geq-1 D S$ or $\geq+2$ DS for mild visual impairment $(\log$ MAR $>0.3$ to $<0.45), \geq-1.5$ DS or $\geq+3$ DS for moderate visual impairment (logMAR 0.45 to $<1.0$ ), $\geq-3$ DS or $\geq+6$ DS for severe visual impairment (logMAR 1.0 to $<1.3$ ) and $\geq-4$ DS or $\geq+8 \mathrm{DS}$ for blindness ( $\log M A R \geq 1.3)$. As the best-corrected visual acuity was not recorded for participants, it was not possible to identify if participants with visual impairment despite habitual correction were also undercorrected. Visual acuity data was incomplete for one eye of 36 cases, and only the visual acuity data for the fellow eye was included.

\section{Statistical analysis}

Descriptive statistics were used to report the prevalence and primary diagnoses leading to visual impairment in the UK Biobank participants. The characteristics of the cases were initially compared with the controls using descriptive statistics, both for all cases and then according to monocular or binocular visual impairment status. Univariate multinomial logistic regression models were used to investigate the association between known risk factors and other predetermined socioeconomic, 
biometric, lifestyle and medical variables and both binocular and monocular visual impairment compared with the matched controls. Those factors found to be associated with visual impairment at a $10 \%$ level (to account for potential correlation between factors) were then included in a multivariable multinomial logistic regression model. Only those that were significantly associated $(\mathrm{P}<0.05)$ with visual impairment remained in the final multivariable model. Although the cases and controls were matched by age, sex and ethnic group, it is recommended also to adjust for these variables to account for any remaining differences; therefore, the final multivariable models included these variables irrespective of their association with visual impairment. ${ }^{11}$ The final multivariable model was checked for colinearity $(r>0.7$ and $\mathrm{P}<0.05$ ) between variables included in the model, and no evidence was found.

\section{RESULTS}

From the 65033 UK Biobank participants with imaging and self-reported eye history, 8359 had mild visual impairment or worse in at least one eye. The majority (6682: $80 \%$ ) had monocular visual impairment, and the degree of visual impairment was typically mild or moderate (online supplementary table 2). As a proportion of all the eligible UK Biobank participants with baseline and extended eye assessments (65 033), the prevalence of monocular and binocular visual impairment was $10.3 \%$ (95\% CI $10.0 \%$ to $10.5 \%$ ) and $2.6 \%$ (95\% CI $2.5 \%$ to $2.7 \%)$, respectively. The prevalence of mild, moderate, severe visual impairment and blindness in the betterseeing eye of those with binocular visual impairment was $1.63 \%$ (95\% CI $1.53 \%$ to $1.73 \%$ ), $0.92 \%$ (95\% CI $0.85 \%$ to $0.99 \%), 0.03 \%$ (95\% CI $0.02 \%$ to $0.04 \%$ ) and $0 \%$, respectively. The estimated prevalence of the same categories in the worse-seeing eye of those with monocular visual impairment was $5.7 \%$ (95\% CI $5.5 \%$ to $5.9 \%$ ), $4.0 \%$ ( $95 \%$ CI $3.8 \%$ to $4.2 \%$ ), $0.57 \%$ (95\% CI $0.51 \%$ to $0.63 \%$ ) and $0.06 \%$ ( $95 \%$ CI $0.04 \%$ to $0.08 \%$ ), respectively.

There were differences in the baseline descriptive statistics in relation to ethnicity, deprivation, employment status, smoking status, medications and self-reported health conditions between the 8359 cases and the 24438 matched controls (online supplementary table 3). For example, $25.5 \%$ of cases lived in the most deprived quintile compared with $21.4 \%$ of the controls. There would also appear to be proportionally more cases with binocular visual impairment living in deprived areas, compared with those with monocular visual impairment. Of those with binocular visual impairment (1677), 30.9\% lived in the most deprived quintile compared with only $24.1 \%$ of those with monocular visual impairment (6682).

The following factors were found to be significantly (at $5 \%$ level) associated with monocular or binocular visual impairment, compared with controls: age, ethnicity, deprivation, employment, systolic blood pressure, waist hip ratio, current smoking status, the presence of a smoker in household, medication for hypertension or hypercholesterolaemia and a history of diabetes mellitus, cardiovascular or cerebrovascular disease (online supplementary table 4). These factors were included in a multivariable model to identify independent factors associated with visual impairment. Sex (as a matching variable) and estimated total retinol daily intake (as associated with the outcome at the $10 \%$ level) were also included in the multivariable model. Only age, ethnicity and deprivation remained significantly (at $5 \%$ level) associated with monocular and binocular visual impairments (online supplementary table 4 ). Those living in the most deprived quintile were $25 \%$ more likely than those in the least deprived quintile to have monocular visual impairment rather than no impairment, and $84 \%$ more likely to have binocular visual impairment, on adjustment for differences in age, sex and ethnic group. With each increasing decade of age, the risk of monocular visual impairment increases by $13 \%$, and the risk of binocular visual impairment increases by $24 \%$, adjusting for ethnic group, sex and deprivation differences. Black and other ethnic participants were $29 \%$ and $53 \%$, respectively, more likely to have binocular visual impairment rather than none, compared with white participants, accounting for age, sex and deprivation differences. However, no significant association was found between ethnicity and monocular visual impairment compared with none, with or without adjustment for age, sex and deprivation differences, indicating that the association between ethnicity and visual impairment may only apply to binocular visual impairment.

For the sample of $25 \%$ of the cases with visual impairment, the primary diagnoses leading to monocular and binocular visual impairments are shown in online supplementary table 5 . In both the better-seeing and worse-seeing eyes of those with binocular visual impairment, the most common identifiable causes were uncorrected refractive error, cataract and vitreoretinal interface disease. For the cases with monocular visual impairment, the most common identifiable causes were cataract, self-reported amblyopia and uncorrected refractive error. No primary cause for the level of visual impairment could be identified for up to $49.8 \%$ of eyes with visual impairment.

The majority of OCT images were of high quality, with the quality of $1836(73.5 \%)$ images rated as good and $550(22.0 \%)$ rated as adequate. The quality of the colour fundus photographs was lower with 1157 (46.3\%) images rated as good, $811(32.5 \%)$ rated as adequate, due to either technical issues (649) or media opacity (162), and $528(21.2 \%)$ images were felt to be of inadequate quality, due to either technical issues (429) or media opacity (99).

\section{DISCUSSION}

For the UK Biobank population with baseline and extended eye data, the prevalence of monocular and binocular visual impairment was $10.3 \%$ and $2.6 \%$, respectively. Visual impairment was associated with increasing age and deprivation and ethnic minority status. Cataract, 
amblyopia and uncorrected refractive error were the most common identifiable causes of visual impairment. However, despite access to self-reported eye history, autorefraction, colour fundus photography and spectral domain OCT imaging, it was not possible to identify the primary cause of visual impairment for almost $50 \%$ of participants.

The prevalence of both binocular and monocular visual impairment in the UK Biobank cohort is lower than for many studies from other industrialised countries. ${ }^{5}$ For an urban Canadian population aged 40 years and over, Robinson et al reported a binocular visual impairment prevalence of $2.7 \% .^{12}$ Among residents of Victoria, Australia, VanNewkirk et $a l^{13}$ recorded that the prevalence of any visual impairment was $4.2 \%$, although both visual acuity and visual field were included in the definition of visual impairment. Similarly, Rahi $e t a l^{14}$ identified binocular visual impairment with habitual correction in $4.5 \%$ of British adults aged 44 or 45 years. However, for the NHANES study population, Vitale $e t$ al reported visual impairment prevalence figures of $4.3 \%$ and $8.8 \%$ among adults aged 40-59 years and aged 60 years and over, respectively, and in a systematic review, Bourne et al estimated the prevalence of moderate visual impairment of worse to be $3.9 \%$ for Western European adults aged over 50 years. ${ }^{15}$ For monocular visual impairment, the British Birth Cohort reported a prevalence of $11.9 \%$ with habitual correction among a comparable group of adults aged 44 or 45 years. ${ }^{14}$ Much higher figures were reported for other studies. The Blue Mountains Eye Study reported prevalence figures of $3.6 \%$ and $8.2 \%$ among adults aged 49-59 years and 60-69 years, respectively. ${ }^{16}$ Nowak et al reported a prevalence of monocular visual impairment of 27.5\% among adults aged 35-97 in Poland. ${ }^{17}$ Much of the difference in the reported prevalence may be explained by differences in the age and sex of the participants in each study, the definitions of visual impairment and by the nature of the studies themselves. ${ }^{18}$

The UK Biobank study used the Townsend score to record deprivation. This index is determined by combining four census variables and can be calculated for any geographical area including postcodes. Increasing deprivation was associated with both monocular and binocular visual impairment among UK Biobank participants. This association has been reported before, both for UK Biobank and other populations. ${ }^{4519}$ However, it is unclear whether increasing deprivation adds to the risk of visual impairment by, for example, delaying access to eye care services, even though such services are often free within the UK NHS or is a consequence of visual impairment and downward social drift. In this study, employment status was associated with both monocular and binocular visual impairments on univariate analysis only. However, Cumberland et al identified an association between visual impairment and a range of economic variables, including employment, income and household structure, in a larger study involving UK Biobank participants. ${ }^{5}$ Given the association between visual impairment and socioeconomic status, initiatives to tackle preventable visual impairment may help to address health inequality.

Black or black British and other (non-white, or Asian or black) ethnicity were also positively associated with binocular visual impairment. Although the number of cases with these ethnicities was small, associations between visual impairment and ethnicity have been described before for both UK and US populations. ${ }^{20} 21$ While glaucoma and diabetic retinopathy are common diagnoses leading to severe visual impairment and blindness in people of black ethnicity, cataract is the more common diagnoses of milder visual impairment in the same population. ${ }^{22}$ Increasing age was also associated with categories of visual impairment, even those cases and controls were matched in 5-year age bands. No significant, independent association was found with the other, predetermined biometric, lifestyle and medical variables. This may reflect the low prevalence of visual impairment due to retinal vascular diseases, such as age-related macular degeneration, diabetic retinopathy and retinal vascular occlusion, in the study population.

For the UK Biobank participants with binocular visual impairment, uncorrected refractive error and cataract were the two most common identifiable diagnoses leading to visual impairment in the better-seeing eye. These two conditions were the largest causes of both mild and moderate visual impairment, but inherited retinal disease and myopic degeneration were the largest causes of severe visual impairment. In contrast to the sight impairment certification data, age-related macular degeneration, glaucoma and diabetic retinopathy were uncommon diagnoses leading to visual impairment. ${ }^{23}$ Shweikh et al reported a $1.7 \%$ prevalence of self-reported glaucoma among UK Biobank participants, but mean visual acuity was good at better than logMAR 0.1 in each eye, so that the contribution of glaucoma to visual impairment based on an acuity definition alone may be smaller than expected. ${ }^{4}$ In a systematic review, Bourne et $a l$ estimated that uncorrected refractive error and cataract were the most common causes of moderate and severe visual impairment in adults in Western Europe, with refractive error accounting for almost $50 \%$ of the visual impairment burden. ${ }^{1}$ Many other studies have also reported that uncorrected refractive error accounts for the majority of visual impairment in adults from developed nations. $^{12-15}$

For monocular visual impairment, cataract, amblyopia and uncorrected refractive error were the most common identifiable diagnoses leading to visual impairment. For moderate or worse visual impairment, self-reported amblyopia was the single most common diagnosis. In the Blue Mountains Eye Study, amblyopia was the most frequent diagnosis leading to mild or worse unilateral visual impairment in persons aged 49-59 years, but cataract was the most common cause of mild unilateral visual impairment in older people. ${ }^{9}{ }^{16}$ Similarly, Nowak et al reported that uncorrected refractive error, amblyopia, cataract and ARMD were the most common causes 
of monocular visual impairment among adults, aged 35-97 years, and data from the British Birth Cohort suggest that refractive error accounted for $43 \%$ of monocular visual impairment. ${ }^{14}{ }^{17}$ By contrast, in the Salisbury Eye Evaluation Study, involving American adults aged 65-84 years, ARMD, other retinal diseases, trauma and amblyopia were the most common causes of monocular blindness. ${ }^{24}$

No primary cause for the recorded level of visual impairment could be identified in almost half of the eyes in the nested study. Visual acuity in the UK Biobank study was recorded using a semiautomated computerised test, based on the EDTRS logMAR chart. Although this test system has not yet been validated within a similar study population, it seems unlikely that there are significant errors in the recording of visual acuity as LogMAR vision charts are considered to be the reference standard for assessing visual function. Self-reported use of either spectacles or contact lenses was over $80 \%$, both for participants with and without visual impairment. ${ }^{18}$ Habitual correction worn at the time of the visual acuity test was assumed to be correct, and uncorrected refractive error was only considered to be a potential cause of visual impairment when either there was no habitual correction or the correction was reported as forgotten. The absence of a best-corrected or pin-hole acuity measure makes it likely that the contribution of uncorrected refractive error may have been underestimated..$^{25} 26$ The absence of lens or anterior segment images and slit-lamp examination also means that underdiagnosis may also apply to cataract, especially as a cause of mild and moderate visual impairment. The inclusion of spectral domain OCT images may have contributed to a higher prevalence of visual impairment due to vitreoretinal interface disease was then expected.

Image quality was generally good, particularly for the OCT images. The inclusion of the OCT images is likely to have aided in the diagnosis of macular oedema and VRIA. ${ }^{27}$ Even when the quality of either image was graded as inadequate, it was often possible to derive some information that could be used for grading, either from the inadequate image itself or the other image. This was most often the case when the fundus photograph was graded as inadequate, but the OCT image was graded as good or adequate. MacGillivray et al also identified problems with the quality of the colour fundus images among UK Biobank participants. ${ }^{28}$

This study has a number of strengths and potential weaknesses. The UK Biobank project was large and had good geographical coverage. It was one of the first to collect spectral domain OCT images and over 65000 participants had baseline and extended eye data, meeting the criteria for inclusion in this study. Many of the key findings in relation to the causes of and associations with visual impairment are consistent with other studies. However, the UK Biobank study is not a population-based study, and the response rate to the invitation to participate was very low. Participants were generally healthier, older, more affluent and more likely to be urban than the full UK population and so may not be representative. ${ }^{35} 1826$ This study also involved only those participants with baseline and enhanced eye assessments. As a result, the visual impairment prevalence figures reported here may not be applicable to the wider UK population and are likely to be minimum prevalence estimates. Furthermore, for the cases with both monocular and binocular visual impairments, no cause was identified in almost $50 \%$ of cases. Habitual correction was assumed to be correct, and this may have underestimated the contribution of refractive error. Similarly, many eyes may have more than one diagnosis leading to visual impairment. Without a single pathology to explain all of the impairment, no diagnosis would be recorded for these eyes, potentially reducing the overall contribution of both refractive error and ocular pathology. Finally, the diagnosis of monocular and binocular amblyopia was also based on the self-reported eye history and normal imaging but did not take account of refractive error. ${ }^{29}$ Limiting the analysis to UK Biobank participants with both baseline and extended eye data may also have caused the study to be underpowered. ${ }^{5}$

The prevalence of visual impairment of any level in the UK Biobank cohort is lower than for population-based studies from other industrialised countries. Although visual impairment was typically mild or moderate, monocular and binocular visual impairment were associated with increasing age and deprivation and ethnic minority status. Cataract and uncorrected refractive error were the most common identifiable causes of both monocular and binocular visual impairments. Despite access to self-reported eye history and both fundus photographs and spectral domain OCT images, no cause was identified for the level of visual impairment recorded in almost half the cases. Therefore, the methodology used in the UK Biobank study may be inadequate to identify the primary causes of visual impairment with confidence. Results from analysis of the UK Biobank dataset may also not be applicable to the wider UK population.

Acknowledgements The authors would like to thank Nicola Dowd, Richard Cannon, Mike Stockton and Grigorios Tzamos for their advice and help with image grading and the creation of the customised grading database. This research has been conducted using the UK Biobank Resource under Application Number 1100.

Collaborators Professor Tariq Aslam, Professor Paul Bishop (Manchester University); Professor Sarah Barman (Kingston University); Professor Jenny Barrett, Dr Sarah Mackie (University of Leeds); Peter Blows, Michelle Chan, Dr Alexander Day, Parul Desai, Dr Cathy Egan, Dan Gore, Anthony Khawaja, Gerassimos Lascaratos, Praveen Patel, Professor Sobha Sivaprasad, Nicholas Strouthidis, Dhanes Thomas, Adnan Tufail, Dr Marta Ugarte, Ananth Viswanathan (Moorfields Eye Hospital, London); Dr Catey Bunce, Professor Chris Hammond, Dr Pirro Hysi, Eoin 0'sullivan, Dr Katie Williams (King's College London); Dr Roxana Carare, Professor Sarah Ennis, Dr Jane Gibson, Srini Goverdhan, Professor Andrew Lotery, Jay Self (University of Southampton); Professor Usha Chakravarthy, Dr Ruth Hogg, Professor Anne Hughes, Michelle Mcgaughey, Dr Bernadette Mcguinness, Dr Gareth Mckay, Euan Paterson, Dr Tunde Peto, Professor Jayne Woodside (Queen's University, Belfast); Antonietta Chianca, Dr Valentina Cipriani, Professor Paul Foster, Dr Marcus Fruttiger, Professor David (ted) Garway-heath, Professor Alison Hardcastle, Pearse A Keane, Professor Peng Tee Khaw, Professor Phil Luthert, Professor Tony Moore, Zaynah Muthy, Dr Caroline Thaung (UCL Institute of Ophthalmology); Professor David Crabb, Dr Haogang Zhu (City University, London); Philippa Cumberland, Professor Jugnoo Rahi (UCL Institute of Child Health); 
Professor Bal Dhillon, Dr Danny Mitry, Dr Tom Macgillivray, Professor Cathie Sudlow, Dr Veronique Vitart(University of Edinburgh); Professor Andrew Dick, Cathy Williams (University of Bristol); Dr John Gallacher (University of Oxford); Professor Jeremy Guggenheim, Professor James Morgan (Cardiff University); Professor Simon Harding, Dr Yalin Zheng (university ofliverpool); Professor Keith Martin, Professor John Yates, Jennifer Yip (University of Cambridge); Dr Chris Owen, Dr Alicja Rudnicka (St George's,University of London); Dr Axel Petzold (UCL Institute of Neurology); Carlota Grossi Sampedro, Dr Max Yates (University of East Anglia); David Steel (Newcastle University); Irene Stratton (Gloucestershire Hospitals NHS Foundation Trust); Professor Emanuele Trucco (University of Dundee); Professor Stephen Vernon (University Hospital, Nottingham). The list of members of the Eye and Vision Consortium is available from the consortium website http://www. ukbiobankeyeconsortium.org.uk/people.

Contributors MM planned the study, secured funding, led the image grading and data analysis and was responsible for the initial draft of the manuscript. DS and TMF have been involved in the study design, data analysis and review of the manuscript. The other members of the UK Biobank Eye and Vision Consortium are listed as collaborators and have had the opportunity to review the data and comment of the data before the manuscript was submitted for publication.

Funding This study was supported as an Investigator Initiated Trial by Alcon Research Ltd.

Competing interests None declared.

Patient consent Detail has been removed from this case description/these case descriptions to ensure anonymity. The editors and reviewers have seen the detailed information available and are satisfied that the information backs up the case the authors are making.

Ethics approval The UK Biobank project received approval from the North-West Multicentre Research Ethics Committee.

Provenance and peer review Not commissioned; externally peer reviewed.

Open Access This is an Open Access article distributed in accordance with the Creative Commons Attribution Non Commercial (CC BY-NC 4.0) license, which permits others to distribute, remix, adapt, build upon this work non-commercially, and license their derivative works on different terms, provided the original work is properly cited and the use is non-commercial. See: http://creativecommons.org/ licenses/by-nc/4.0/

(C) Article author(s) (or their employer(s) unless otherwise stated in the text of the article) 2018. All rights reserved. No commercial use is permitted unless otherwise expressly granted.

\section{REFERENCES}

1. Bourne RR, Jonas JB, Flaxman SR, et al. Prevalence and causes of vision loss in high-income countries and in Eastern and Centra Europe: 1990-2010. Br J Ophthalmol 2014;98:629-38.

2. Sudlow C, Gallacher J, Allen N, et al. UK biobank: an open access resource for identifying the causes of a wide range of complex diseases of middle and old age. PLoS Med 2015;12:e1001779.

3. Cumberland PM, Bao Y, Hysi PG, et al. Frequency and distribution of refractive error in adult life: methodology and findings of the UK biobank study. PLoS One 2015;10:e0139780.

4. Shweikh Y, Ko F, Chan MP, et al. Measures of socioeconomic status and self-reported glaucoma in the U.K. Biobank cohort. Eye 2015;29:1360-7.

5. Cumberland PM, Rahi JS. UK Biobank Eye and Vision Consortium. Visual function, social position, and health and life chances: the UK biobank study. JAMA Ophthalmol 2016;134:959-66

6. Stalmans P, Duker JS, Kaiser PK, et al. Oct-based interpretation of the vitreomacular interface and indications for pharmacologic vitreolysis. Retina 2013;33:2003-11.

7. Wilkinson CP, Ferris FL, Klein RE, et al. Proposed international clinical diabetic retinopathy and diabetic macular edema disease severity scales. Ophthalmology 2003;110:1677-82.
8. Bird AC, Bressler NM, Bressler SB, et al. An international classification and grading system for age-related maculopathy and age-related macular degeneration. The International ARM Epidemiological Study Group. Surv Ophthalmol 1995;39:367-74.

9. Attebo K, Mitchell P, Cumming R, et al. Prevalence and causes of amblyopia in an adult population. Ophthalmology 1998;105:154-9.

10. Foster PJ, Buhrmann R, Quigley HA, et al. The definition and classification of glaucoma in prevalence surveys. $\mathrm{Br} \mathrm{J} \mathrm{Ophthalmol}$ 2002;86:238-42.

11. Pearce N. Analysis of matched case-control studies. BMJ 2016;352:i969.

12. Robinson $B$, Feng $Y$, Woods $C A$, et al. Prevalence of visual impairment and uncorrected refractive error - report from a Canadian urban population-based study. Ophthalmic Epidemiol 2013;20:123-30.

13. VanNewkirk MR, Weih L, McCarty CA, et al. Cause-specific prevalence of bilateral visual impairment in Victoria, Australia: the Visual Impairment Project. Ophthalmology 2001:108:960-7.

14. Rahi JS, Cumberland PM, Peckham CS. Visual impairment and vision-related quality of life in working-age adults: findings in the 1958 British birth cohort. Ophthalmology 2009;116:270-4.

15. Vitale S, Cotch MF, Sperduto RD. Prevalence of visual impairment in the United States. JAMA 2006;295:2158-63.

16. Wang JJ, Foran S, Mitchell P. Age-specific prevalence and causes of bilateral and unilateral visual impairment in older Australians: the Blue Mountains Eye Study. Clin Exp Ophthalmol 2000;28:268-73.

17. Nowak MS, Smigielski J. The prevalence of age-related eye diseases and cataract surgery among older adults in the city of Lodz, Poland. $J$ Ophthalmol 2015;2015:1-7.

18. Dawes P, Dickinson C, Emsley R, et al. Vision impairment and dual sensory problems in middle age. Ophthalmic Physiol Opt 2014;34:479-88.

19. Chou CF, Beckles GL, Zhang X, et al. Association of socioeconomic position with sensory impairment among US working-aged adults. Am J Public Health 2015;105:1262-8.

20. Sivaprasad S, Gupta B, Gulliford MC, et al. Ethnic variation in the prevalence of visual impairment in people attending diabetic retinopathy screening in the United Kingdom (DRIVE UK). PLoS One 2012;7:e39608

21. Varma R, Vajaranant TS, Burkemper B, et al. Visual impairment and blindness in adults in the United States: demographic and geographic variations from 2015 to 2050. JAMA Ophthalmol 2016;134:802-9.

22. Congdon N, O'Colmain B, Klaver CC, et al. Causes and prevalence of visual impairment among adults in the United States. Arch Ophthalmol 2004;122:477-85.

23. Mitry D, Bunce C, Wormald R, et al. Causes of certifications for severe sight impairment (blind) and sight impairment (partial sight) in children in England and Wales. Br J Ophthalmol 2013;97:1431-6.

24. Muñoz B, West SK, Rubin GS, et al. Causes of blindness and visual impairment in a population of older Americans: The Salisbury Eye Evaluation Study. Arch Ophthalmol 2000;118:819-25.

25. Taylor HR, Livingston PM, Stanislavsky YL, et al. Visual impairment in Australia: distance visual acuity, near vision, and visual field findings of the Melbourne Visual Impairment Project. Am J Ophthalmol 1997;123:328-37.

26. Machan CM, Lillakas L, Hrynchak PK, et al. Eye examinations improve visual acuity across ages. Can J Ophthalmol 2013;48:286-91.

27. Meuer SM, Myers CE, Klein BE, et al. The epidemiology of vitreoretinal interface abnormalities as detected by spectraldomain optical coherence tomography: the beaver dam eye study. Ophthalmology 2015;122:787-95

28. MacGillivray TJ, Cameron JR, Zhang Q, et al. Suitability of UK biobank retinal images for automatic analysis of morphometric properties of the vasculature. PLoS One 2015;10:e0127914.

29. Xiao O, Morgan IG, Ellwein LB, et al. Prevalence of amblyopia in school-aged children and variations by age, gender, and ethnicity in a multi-country refractive error study. Ophthalmology 2015;122:1924-31. 